\title{
Global existence for quasilinear diffusion equations in isotropic nondivergence form
}

\author{
WolfGANG ARENDT AND RALPH CHILL
}

Dedicated to Herbert Amann on the occasion of his 70th birthday

\begin{abstract}
We consider the quasilinear parabolic equation

$$
u_{t}-\beta(t, x, u, \nabla u) \Delta u=f(t, x, u, \nabla u)
$$

in a cylindrical domain, together with initial-boundary conditions, where the quasilinearity operates on the diffusion coefficient of the Laplacian. Under suitable conditions we prove global existence of a solution in the energy space. Our proof depends on maximal regularity of a nonautonomous linear parabolic equation which we use to provide us with compactness in order to apply Schaefer's fixed point theorem.
\end{abstract}

Mathematics Subject Classification (2010): 35K15 (primary); 35A05, 35K55 (secondary).

\section{Introduction}

We prove global existence of a solution of the quasilinear diffusion problem

$$
\begin{cases}u_{t}-\beta(t, x, u, \nabla u) \Delta u=f(t, x, u, \nabla u) & \text { in }(0, \infty) \times \Omega, \\ u=0 & \text { in }(0, \infty) \times \partial \Omega, \\ u(0, \cdot)=u_{0}(\cdot) & \text { in } \Omega,\end{cases}
$$

where $\Omega \subset \mathbb{R}^{d}$ is an open set, $u_{0} \in H_{0}^{1}(\Omega)$ and

$$
\begin{aligned}
& \beta:(0, \infty) \times \Omega \times \mathbb{R}^{1+d} \rightarrow\left[\varepsilon, \frac{1}{\varepsilon}\right] \quad(\varepsilon \in(0,1) \text { is fixed }) \text { and } \\
& f:(0, \infty) \times \Omega \times \mathbb{R}^{1+d} \rightarrow \mathbb{R}
\end{aligned}
$$

are measurable functions which are continuous with respect to the last variable, for every $(t, x) \in(0, \infty) \times \Omega$. The function $f$ satisfies in addition a linear growth condition with respect to the last variable.

Received November 25, 2008; accepted in revised form July 27, 2009. 
We prove in fact existence of a solution in the space

$$
H_{\mathrm{loc}}^{1}\left([0, \infty) ; L^{2}(\Omega)\right) \cap L_{\mathrm{loc}}^{2}\left([0, \infty) ; D\left(\Delta_{D}\right)\right) \cap C\left([0, \infty) ; H_{0}^{1}(\Omega)\right),
$$

where $D\left(\Delta_{D}\right)$ is the domain of the Dirichlet-Laplacian in $L^{2}(\Omega)$.

Note that this existence result is also a maximal regularity result. Maximal regularity of the abstract linear inhomogeneous problem

$$
\dot{u}(t)+A u(t)=f(t) \text { for a.e. } t \in(0, T), \quad u(0)=0,
$$

has obtained much attention in recent years. Given a closed linear operator $A$ on $L^{2}(\Omega)$ (we will only consider the $L^{2}$ setting here), saying that this problem has maximal regularity means that for every $f \in L^{2}\left(0, T ; L^{2}(\Omega)\right)$ there exists a unique solution in the maximal regularity space

$$
M R:=H^{1}\left(0, T ; L^{2}(\Omega)\right) \cap L^{2}(0, T ; D(A))
$$

in particular, the two terms on the left-hand side of the above differential equation have the same regularity as the inhomogeneity $f$.

It is known that maximal regularity results can be applied to solve non-linear problems by using fixed point theorems. Mostly, if some Lipschitz continuity is available (for example by making appropriate assumptions on the regularity and the growth of the coefficients $\beta$ and $f$ ), then Banach's fixed point theorem is used to establish local existence; see, for example, [1,2, 4, 5], [12, Chapters 7 and 8], [13]. On the other hand, if come compactness is available (for example by assuming that $\Omega$ is bounded and regular), Schauder's fixed point theorem for continuous mappings on Banach spaces can be used in order to establish existence of solutions; see $[10,11]$.

We follow the second way but we will make no assumptions on boundedness or regularity of the set $\Omega$, nor will we impose further regularity of the coefficients $\beta$ and $f$. We will instead use that the injection of $M R=H^{1}\left(0, T ; L^{2}(\Omega)\right) \cap$ $L^{2}\left(0, T ; D\left(\Delta_{D}\right)\right)$ into $L^{2}\left(0, T ; H_{\text {loc }}^{1}(\Omega)\right)$ is compact by local regularity results for the Laplace operator, by Rellich's theorem and by a result of Aubin-Lions. This will allow us to use versions of Schauder's fixed point theorem in Fréchet spaces instead of Banach spaces. Most useful for our purposes is Schaefer's fixed point theorem which replaces invariance of a convex set by an a priori estimate. Section 2 is devoted to recalling this fixed point theorem which can be even formulated in complete locally convex spaces thanks to Tychonov's version of Schauder's fixed point theorem.

ACKNOWLEDGEMENTS. The authors are most grateful to Eva Fašangová for a stimulating discussion on maximal regularity of the nonautonomous linear problem. 


\section{Schaefer's fixed point theorem}

In a short article in Mathematische Annalen from 1955, Schaefer gave an elegant proof of a result from Leray-Schauder theory which is most suitable for applications in partial differential equations, [14, Satz]. This proof is reproduced in several textbooks and frequently cited as Schaefer's Fixed Point Theorem; see, for example, [8]. But Schaefer also gave an extension of this fixed point theorem to complete locally convex spaces. It turns out that, when proving existence of a solution of (1.1), we will encounter a situation where this is useful. The reason is that some compact embedding is needed. If $\Omega \subset \mathbb{R}^{d}$ is an open set, then the embedding $H^{2}(\Omega) \hookrightarrow H^{1}(\Omega)$ is compact if $\Omega$ is a bounded Lipschitz domain, but in general not if $\Omega$ is unbounded or the boundary is bad. However, the embedding $H_{\text {loc }}^{2}(\Omega) \hookrightarrow$ $H_{\text {loc }}^{1}(\Omega)$ is compact for arbitrary open sets.

Schaefer deduces by a simple argument his fixed point theorem from Schauder's fixed point theorem in the case of a Banach space, and from Tychonov's fixed point theorem [16] in the case of a complete locally convex space.

We take the opportunity to reformulate Schaefer's fixed point theorem in such generality, choosing a formulation which makes it directly applicable in our context. This result is the precise setting where the philosophy that an a priori bound of the solution implies the existence of the solution becomes truth. It is a consequence of the following extension of Schauder's fixed point theorem due to Tychonov.

Theorem 2.1. (Schauder's fixed point theorem in locally convex vector space, [16]). Let $E$ be a complete locally convex vector space, $\mathcal{C}$ a nonempty, convex subset of $E$ and $T: \mathcal{C} \rightarrow \mathcal{C}$ a continuous mapping. If $T \mathcal{C}$ is contained in a compact subset of $\mathcal{C}$, then $T$ has a fixed point.

Theorem 2.2 (Schaefer's fixed point theorem). Let $E$ be a complete locally convex vector space and let $T: E \rightarrow E$ be a continuous mapping. Assume that there exist a continuous seminorm $p: E \rightarrow \mathbb{R}_{+}$, a constant $R>0$ and a compact set $\mathcal{K} \subset E$ such that the Schaefer set

$$
\mathcal{S}:=\{u \in E: u=\lambda T u \text { for some } \lambda \in[0,1]\}
$$

is included in

$$
\mathcal{C}:=\{u \in E: p(u)<R\}
$$

and such that $T \mathcal{C} \subset \mathcal{K}$. Then $T$ has a fixed point.

Proof. Define $\widetilde{T}: \overline{\mathcal{C}} \rightarrow \overline{\mathcal{C}}(\overline{\mathcal{C}}$ being the closure of $\mathcal{C})$ by

$$
\widetilde{T} u:= \begin{cases}T u & \text { if } p(T u) \leq R \\ \frac{R}{p(T u)} T u & \text { if } p(T u)>R .\end{cases}
$$

Then $\widetilde{T}$ is continuous and $\widetilde{T} \overline{\mathcal{C}} \subset[0,1] \cdot \mathcal{K}$. The set $[0,1] \times \mathcal{K}$ is compact by Tychonov's theorem and thus $[0,1] \cdot \mathcal{K}$ is compact as the continuous image of $[0,1] \times \mathcal{K}$ 
for the mapping $(\lambda, u) \mapsto \lambda \cdot u$. It follows from Theorem 2.1 that $\widetilde{T}$ has a fixed point $u \in \mathcal{C}$. By definition of $\widetilde{T}, u=\widetilde{T} u=\lambda T u$ for some $\lambda \in[0,1]$, that is $u \in \mathcal{S}$.

Note that $\lambda<1$ if and only if $p(T u)>R$, and in that case $p(\widetilde{T} u)=R$. However, since $\mathcal{S}$ is included in $\mathcal{C}$, we have $p(\widetilde{T} u)=p(u)<R$. Hence, $\lambda=1$ and $u$ is a fixed point of $T$.

\section{The linear problem}

Let $\Omega \subset \mathbb{R}^{d}$ be an open set. Let $V$ be a Hilbert space which embeds densely and continuously into $L^{2}(\Omega)$ (we write $V \hookrightarrow L^{2}(\Omega)$ ) and let $a: V \times V \rightarrow \mathbb{R}$ be a bilinear, symmetric form. We assume throughout that $a$ is bounded and $L^{2}(\Omega)$ elliptic, which means, respectively,

$$
\begin{aligned}
& |a(u, v)| \leq M\|u\|_{V}\|v\|_{V} \text { for some } M \geq 0 \text { and all } u, v \in V, \text { and } \\
& a(u)+\omega\|u\|_{L^{2}}^{2} \geq \eta\|u\|_{V}^{2} \text { for some } \omega \geq 0, \eta>0 \text { and all } u \in V .
\end{aligned}
$$

Here and in the following we shortly write $a(u)$ for $a(u, u)$.

Denote by $A$ the operator associated with $a$ on $L^{2}(\Omega)$, that is, for $u, f \in L^{2}$ one has $u \in D(A)$ and $A u=f$ if and only if $u \in V$ and $a(u, v)=(f, v)_{L^{2}}$ for every $v \in V$. The operator $A$ is closed and $D(A)$, when equipped with the graph norm, is a Banach space.

Then the following maximal regularity result is well known: for all $f \in$ $L^{2}\left(0, T ; L^{2}(\Omega)\right), u_{0} \in V$, there exists a unique solution of the autonomous problem

$$
\begin{aligned}
& u \in H^{1}\left(0, T ; L^{2}(\Omega)\right) \cap L^{2}(0, T ; D(A)), \\
& u(t)+A u(t)=f(t) \quad \text { for almost every } t \in(0, T), \\
& u(0)=u_{0} .
\end{aligned}
$$

Recall that the maximal regularity space

$$
M R:=H^{1}\left(0, T ; L^{2}(\Omega)\right) \cap L^{2}(0, T ; D(A))
$$

which is equipped with the norm

$$
\|u\|_{M R}^{2}:=\int_{0}^{T}\|u(t)\|_{L^{2}}^{2}+\int_{0}^{T}\|\dot{u}(t)\|_{L^{2}}^{2}+\int_{0}^{T}\|A u(t)\|_{L^{2}}^{2}
$$

is continuously embedded in $C([0, T] ; V)$, [7, Exemple 1, page 577]. We will need the following product rule; see [7, Théorème 2, page 575] for a similar result.

Lemma 3.1. Let $u \in M R$. Then $a(u(\cdot)) \in W^{1,1}(0, T)$ and

$$
\frac{d}{d t} a(u(t))=2(A u(t), \dot{u}(t))_{L^{2}} \quad \text { for almost every } t \in(0, T) .
$$


Proof. For $u \in C^{1}([0, T] ; D(A))$, the assertion is a consequence of the product rule, the symmetry of the form $a$, and the definition of the operator $A$ :

$$
\frac{d}{d t} a(u(t))=2 a(u(t), \dot{u}(t))=2(A u(t), \dot{u}(t))_{L^{2}} .
$$

For arbitrary $u \in M R$, the assertion follows from this and an approximation by functions in $C^{1}([0, T] ; D(A))$. The fact that $C^{1}([0, T] ; D(A))$ is dense in $M R$ follows from classical techniques using regularization; compare with [7, Lemme 4 , page 586].

Now we consider a new problem, obtained by a multiplicative perturbation.

Theorem 3.2 (Linear, nonautonomous problem). Let $m:(0, T) \times \Omega \rightarrow\left[\varepsilon, \frac{1}{\varepsilon}\right]$ be a measurable function, where $\varepsilon \in(0,1)$ is fixed. Then, for every $f \in L^{2}\left(0, T ; L^{2}(\Omega)\right)$, $u_{0} \in V$ there exists a unique solution of the problem

$$
\begin{aligned}
& u \in M R=H^{1}\left(0, T ; L^{2}(\Omega)\right) \cap L^{2}(0, T ; D(A)) \\
& \dot{u}(t)+m(t, \cdot) A u(t)=f(t) \quad \text { for almost every } t \in(0, T), \\
& u(0)=u_{0} .
\end{aligned}
$$

Moreover, there exists a constant $c=c\left(\varepsilon, M, \eta, \omega, c_{1}, T,\right) \geq 0\left(c_{1}\right.$ being the embedding constant of the embedding $V \hookrightarrow L^{2}(\Omega)$ ) independent of $f$ and $u_{0}$ such that

$$
\|u\|_{M R} \leq c\left(\|f\|_{L^{2}\left(0, T ; L^{2}(\Omega)\right)}+\left\|u_{0}\right\|_{V}\right),
$$

for each solution u of (3.4).

Remark 3.3. The constant $c$ in (3.5) depends on the constants $\varepsilon, M, \eta, \omega, c_{1}$ and the time $T$, but it does not depend on other properties of the form $a$ or the function $m$.

Remark 3.4. For many concrete operators $A$, problem (3.4) is a particular case of the parabolic equation

$$
u_{t}-\sum_{i, j} a_{i j}(t, x) \partial_{i j} u=f(t, x) \quad \text { in }(0, T) \times \Omega
$$

equipped with initial-boundary conditions, where the coefficients $a_{i j}$ belong to $L^{\infty}((0, T) \times \Omega)$. For this equation and if $\Omega$ satisfies a weak regularity condition, it might be possible to prove existence of weak and bounded solutions for bounded initial data and right-hand sides $f \in L^{d+1}((0, T) \times \Omega)$ by means of a general parabolic maximum principle [17] (see [3] for the elliptic version). This method would be completely different from ours, but it is not clear whether one can obtain $L^{2}$-maximal regularity in $L^{2}(\Omega)$ in this way. The problem of $L^{q}$-maximal regularity in $L^{p}(\Omega)$ is, to our knowledge, open. 
Proof of Theorem 3.2. We use the method of continuity. For every $s \in[0,1]$, consider the function

$$
m_{s}:=(1-s)+s m:(0, T) \times \Omega \rightarrow\left[\varepsilon, \frac{1}{\varepsilon}\right]
$$

and the bounded operator

$$
B_{s}: M R \rightarrow L^{2}\left(0, T ; L^{2}(\Omega)\right) \times V
$$

given by

$$
B_{s} u=\left(\dot{u}+m_{s} A u, u(0)\right) .
$$

Then $B:[0,1] \rightarrow \mathcal{L}\left(M R, L^{2}\left(0, T ; L^{2}(\Omega)\right) \times V\right)$ is continuous and $B_{0}$ is invertible by the maximal regularity result for the autonomous problem (3.3). Thus, in order to prove the theorem, by [9, Theorem 5.2], it suffices to prove the a priori estimate

$$
\begin{aligned}
& \|u\|_{M R} \leq c\left\|B_{S} u\right\|=c\left(\left\|\dot{u}+m_{s} A u\right\|_{L^{2}\left(0, T ; L^{2}(\Omega)\right)}+\|u(0)\|_{V}\right) \\
& \text { for all } s \in[0,1] \text { and all } u \in M R
\end{aligned}
$$

which, for $s=1$, is exactly the estimate (3.5) to be proved.

Let $s \in[0,1]$. Let $u \in M R$ be such that

$$
\dot{u}+m_{s} A u=f \text { and } u(0)=u_{0} .
$$

Then, for almost every $t \in[0, T]$,

$$
\int_{\Omega} \dot{u}(t)^{2} \frac{d x}{m_{s}}+\int_{\Omega} A u(t) \dot{u}(t) d x=\int_{\Omega} f(t) \dot{u}(t) \frac{d x}{m_{s}} .
$$

We recall from Lemma 3.1 that $a(u(\cdot)) \in W^{1,1}$ and $\frac{1}{2} \frac{d}{d t} a(u(t))=(A u(t), \dot{u}(t))_{L^{2}}$ for almost every $t \in(0, T)$. This identity and the Cauchy-Schwarz inequality applied to the term on the right-hand side of the above equality imply that, for almost every $t \in[0, T]$,

$$
\frac{1}{2} \int_{\Omega} \dot{u}(t)^{2} \frac{d x}{m_{s}}+\frac{1}{2} \frac{d}{d t} a(u(t)) \leq \frac{1}{2} \int_{\Omega} f(t)^{2} \frac{d x}{m_{s}} .
$$

Integrating this inequality on $(0, t)$ and using the estimate $\varepsilon \leq \frac{1}{m_{s}} \leq \frac{1}{\varepsilon}$, it follows that

$$
\varepsilon \int_{0}^{t}\|\dot{u}(s)\|_{L^{2}}^{2} d s+a(u(t)) \leq a\left(u_{0}\right)+\frac{1}{\varepsilon} \int_{0}^{T}\|f(s)\|_{L^{2}}^{2} d s .
$$

Thus, by boundedness and ellipticity of the form $a$,

$$
\varepsilon \int_{0}^{t}\|\dot{u}(s)\|_{L^{2}}^{2} d s+\eta\|u(t)\|_{V}^{2} \leq M\left\|u_{0}\right\|_{V}^{2}+\frac{1}{\varepsilon}\|f\|_{L^{2}\left(0, T ; L^{2}(\Omega)\right)}^{2}+\omega\|u(t)\|_{L^{2}}^{2} .
$$


This estimate and the estimate

$$
\begin{aligned}
\|u(t)\|_{L^{2}}^{2} & =\left\|u_{0}\right\|_{L^{2}}^{2}+\int_{0}^{t} \frac{d}{d s}\|u(s)\|_{L^{2}}^{2} d s=\left\|u_{0}\right\|_{L^{2}}^{2}+2 \int_{0}^{t}\langle u(s), \dot{u}(s)\rangle_{L^{2}} d s \\
& \leq\left\|u_{0}\right\|_{L^{2}}^{2}+\frac{2 \omega}{\varepsilon} \int_{0}^{t}\|u(s)\|_{L^{2}}^{2} d s+\frac{\varepsilon}{2 \omega} \int_{0}^{t}\|\dot{u}(s)\|_{L^{2}}^{2} d s
\end{aligned}
$$

yield the estimate

$$
\begin{aligned}
\frac{\varepsilon}{2} \int_{0}^{t}\|\dot{u}(s)\|_{L^{2}}^{2} d s & +\eta\|u(t)\|_{V}^{2} \leq\left(M+\omega c_{1}^{2}\right)\left\|u_{0}\right\|_{V}^{2} \\
& +\frac{1}{\varepsilon}\|f\|_{L^{2}\left(0, T ; L^{2}(\Omega)\right)}^{2}+\frac{2 \omega^{2} c_{1}^{2}}{\varepsilon} \int_{0}^{t}\|u(s)\|_{V}^{2} d s
\end{aligned}
$$

where $c_{1}$ is the embedding constant of the embedding $V \hookrightarrow L^{2}(\Omega)$. From this inequality and Gronwall's lemma it follows that there is a constant $c=c(\varepsilon, M, \eta$, $\left.\omega, c_{1}, T\right) \geq 0$ such that

$$
\sup _{t \in[0, T]}\|u(t)\|_{V}^{2} \leq c\left(\left\|u_{0}\right\|^{2}+\|f\|_{L^{2}\left(0, T ; L^{2}(\Omega)\right)}^{2}\right) .
$$

Inserting this estimate into (3.8), we find that there exists a constant $c=c(\varepsilon, M$, $\left.\eta, \omega, c_{1}, T\right) \geq 0$ (possibly different from the preceding one) such that

$$
\int_{0}^{T}\|\dot{u}(s)\|_{L^{2}}^{2} d s \leq c\left(\left\|u_{0}\right\|^{2}+\|f\|_{L^{2}\left(0, T ; L^{2}(\Omega)\right)}^{2}\right) .
$$

This gives the estimate (3.6) for the second part of the $M R$ norm of $u$. Since $u(t)=u(0)+\int_{0}^{t} \dot{u}(s) d s$, it follows that

$$
\int_{0}^{T}\|u(t)\|_{L^{2}}^{2} d t \leq c\left(\left\|u_{0}\right\|_{V}^{2}+\int_{0}^{T}\|\dot{u}(t)\|_{L^{2}}^{2} d t\right)
$$

for some $c=c\left(c_{1}, T\right) \geq 0$. This gives the estimate for the first part of the $M R$ norm of $u$. Since

$$
\int_{0}^{T}\|A u(t)\|_{L^{2}}^{2} d t \leq \frac{1}{\varepsilon^{2}} \int_{0}^{T}\left\|m_{S} A u(t)\right\|_{L^{2}}^{2} d t
$$

and $m_{s} A u(t)=-\dot{u}(t)+f(t)$, also the third term of the $M R$ norm of $u$ can be estimated, and the proof of (3.6) is complete. 


\section{The nonlinear problem}

Let $\Omega \in \mathbb{R}^{d}$ be an open set. Let $V$ be a closed subspace of $H^{1}(\Omega)$ which is dense in $L^{2}(\Omega)$. We assume for simplicity that $V$ is equipped with the $H^{1}$ norm.

Let $a: V \times V \rightarrow \mathbb{R}$ be a bilinear, symmetric, bounded, $L^{2}$-elliptic form, and denote by $A$ the operator associated with $a$ on $L^{2}(\Omega)$. We assume that

$$
D(A) \subset H_{\mathrm{loc}}^{2}(\Omega) .
$$

Below, in Section 5, we will give several concrete examples for which this condition is satisfied. We consider $D(A)$ with the graph norm and let $M R=H^{1}\left(0, T ; L^{2}(\Omega)\right)$ $\cap L^{2}(0, T ; D(A))$, as in Section 3.

Theorem 4.1. Let $\varepsilon \in(0,1)$ and let

$$
\begin{aligned}
& \beta:(0, T) \times \Omega \times \mathbb{R}^{1+d} \rightarrow\left[\varepsilon, \frac{1}{\varepsilon}\right] \text { be a measurable function such that } \\
& \beta(t, x, \cdot): \mathbb{R}^{1+d} \rightarrow\left[\varepsilon, \frac{1}{\varepsilon}\right] \quad \text { is continuous for almost every }(t, x) .
\end{aligned}
$$

Let moreover

$$
\begin{aligned}
& f:(0, T) \times \Omega \times \mathbb{R}^{1+d} \rightarrow \mathbb{R} \text { be a measurable function such that } \\
& f(t, x, \cdot): \mathbb{R}^{1+d} \rightarrow \mathbb{R} \text { is continuous for almost every }(t, x) \text { and } \\
& |f(t, x, u, p)| \leq g(t, x)+L(|u|+|p|) \text { for every }(t, x, u, p), \text { and some } \\
& g \in L^{2}\left(0, T ; L^{2}(\Omega)\right) \text { and } L \geq 0 .
\end{aligned}
$$

Then, for every $u_{0} \in V$ there exists a solution of the problem

$$
\begin{aligned}
& u \in M R=H^{1}\left(0, T ; L^{2}(\Omega)\right) \cap L^{2}(0, T ; D(A)) \\
& \dot{u}(t)+\beta(t, x, u, \nabla u) A u(t)=f(t, x, u, \nabla u) \text { for almost every } t \in(0, T), \\
& u(0)=u_{0} .
\end{aligned}
$$

Moreover, there exists a constant $c=c(\varepsilon, M, \eta, \omega, L, T) \geq 0$ such that for every solution $u$ of (4.2) one has

$$
\|u\|_{M R} \leq c\left(\left\|u_{0}\right\|_{V}+\|g\|_{L^{2}\left(0, T ; L^{2}(\Omega)\right)}\right) .
$$

Remark 4.2. Under the hypotheses of Theorem 4.1, one may in general not expect uniqueness of solutions. A simple counterexample is given in Example 5.2 below.

Let $\left(\Omega_{k}\right)_{k}$ be an increasing sequence of open, bounded subsets of $\mathbb{R}^{d}$ which are of class $C^{\infty}$ and such that $\bar{\Omega}_{k} \subset \Omega$ and $\bigcup_{k \in \mathbb{N}} \Omega_{k}=\Omega$. Such a sequence $\left(\Omega_{k}\right)_{k}$ exists for every open set $\Omega \subset \mathbb{R}^{d}$; compare with [6, Lemma 1, page 409]. We consider the space

$$
\begin{aligned}
E & :=L^{2}\left(0, T ; H_{\mathrm{loc}}^{1}(\Omega)\right) \\
& :=\left\{u \in L_{\mathrm{loc}}^{2}((0, T) \times \Omega):\left.u\right|_{(0, T) \times \Omega_{k}} \in L^{2}\left(0, T ; H^{1}\left(\Omega_{k}\right)\right) \text { for every } k \in \mathbb{N}\right\},
\end{aligned}
$$


which is a Fréchet space for the sequence $\left(p_{k}\right)$ of seminorms given by

$$
p_{k}(u)^{2}:=\int_{0}^{T} \int_{\Omega_{k}}\left(|u(t, x)|^{2}+|\nabla u(t, x)|^{2}\right) d x d t=\|u\|_{L^{2}\left(0, T ; H^{1}\left(\Omega_{k}\right)\right)}^{2} .
$$

We recall that for an open, bounded set $U \subset \mathbb{R}^{d}$ of class $C^{\infty}$ the injection of $H^{2}(U)$ into $H^{1}(U)$ is compact by Rellich's theorem. As a consequence, the embedding

$$
H^{1}\left(0, T ; L^{2}(U)\right) \cap L^{2}\left(0, T ; H^{2}(U)\right) \hookrightarrow L^{2}\left(0, T ; H^{1}(U)\right)
$$

is compact by a result of Lions-Aubin (see [15, III.1, Proposition 1.3, page 106]). Since $D(A) \subset H_{\text {loc }}^{2}(\Omega)$ by our standing assumption (4.1), and since this embedding is continuous by the closed graph theorem, it follows from the preceding that the embedding

$$
M R=H^{1}\left(0, T ; L^{2}(\Omega)\right) \cap L^{2}(0, T ; D(A)) \hookrightarrow L^{2}\left(0, T ; H_{\mathrm{loc}}^{1}(\Omega)\right)=E
$$

is compact, too.

Remark 4.3. Following the above arguments, it turns out that, in fact, the embedding (4.4) is compact as soon as the embedding

$$
D(A) \hookrightarrow H_{\mathrm{loc}}^{1}(\Omega)
$$

is compact. Compactness of this embedding is ensured by the assumption (4.1), but we do not know whether it is true in general.

Proof of Theorem 4.1. Fix $u_{0} \in V$.

In the first step of the proof we show that for every $k$ the problem

$$
\begin{aligned}
& u \in M R=H^{1}\left(0, T ; L^{2}(\Omega)\right) \cap L^{2}(0, T ; D(A)) \\
& \dot{u}(t)+\beta(t, x, u, \nabla u) A u(t)=f(t, x, u, \nabla u) 1_{\Omega_{k}}(x) \text { for a.e. } t \in(0, T), \\
& u(0)=u_{0}
\end{aligned}
$$

admits a solution and that there exists a constant $c=c(M, \varepsilon, \eta, \omega, L, T) \geq 0$ independent of $k(!)$ such that for every solution of this problem one has

$$
\|u\|_{M R}^{2} \leq c\left(\left\|u_{0}\right\|_{V}^{2}+\|g\|_{L^{2}\left(0, T ; L^{2}(\Omega)\right)}^{2}\right) .
$$

Fix $k \in \mathbb{N}$. For every $v \in E$ we put

$$
\begin{aligned}
m_{v}(t, x) & :=\beta(t, x, v(t, x), \nabla v(t, x)) \quad \text { and } \\
f_{v, k}(t, x) & :=f(t, x, v(t, x), \nabla v(t, x)) 1_{\Omega_{k}}(x) .
\end{aligned}
$$


Then $m_{v}$ and $f_{v, k}$ are measurable functions on $(0, T) \times \Omega, m_{v}$ takes values in $\left[\varepsilon, \frac{1}{\varepsilon}\right]$, and

$$
\begin{aligned}
\left\|f_{v, k}\right\|_{L^{2}\left(0, T ; L^{2}(\Omega)\right)}^{2} & \leq c \int_{0}^{T} \int_{\Omega_{k}}\left[g(t, x)^{2}+\left(|v|^{2}+|\nabla v|^{2}\right)\right] \\
& \leq c\left(\|g\|_{L^{2}\left(0, T ; L^{2}(\Omega)\right)}^{2}+p_{k}(v)^{2}\right)<\infty
\end{aligned}
$$

for some constant $c=c(L) \geq 0$.

Hence, by Theorem 3.2, there exists a unique solution $u=: T_{k} v \in M R$ of the problem

$$
\begin{aligned}
& \dot{u}(t)+m_{v}(t, \cdot) A u(t)=f_{v, k}(t) \text { for almost every } t \in(0, T), \text { and } \\
& u(0)=u_{0} .
\end{aligned}
$$

and there exists a constant $c=c(\varepsilon, M, \eta, \omega, T) \geq 0$ (depending also on the embedding constant of the embedding $V \hookrightarrow L^{2}(\Omega)$, which is now equal to 1) such that

$$
\begin{aligned}
\|u\|_{M R}^{2} & \leq c\left(\left\|u_{0}\right\|_{V}^{2}+\left\|f_{v, k}\right\|_{L^{2}\left(0, T ; L^{2}(\Omega)\right)}^{2}\right) \\
& \leq c\left(\left\|u_{0}\right\|_{V}^{2}+\|g\|_{L^{2}\left(0, T ; L^{2}(\Omega)\right)}^{2}+\|v\|_{L^{2}\left(0, T ; H^{1}\left(\Omega_{k}\right)\right)}^{2}\right) .
\end{aligned}
$$

In this way, we defined an operator $T_{k}: E \rightarrow E$.

(a) We show that $T_{k}$ is continuous. Let $v_{n} \rightarrow v$ in $E$, and let $u_{n}=T_{k} v_{n}$ and $u=T_{k} v$. We have to show that $u_{n} \rightarrow u$ in $E$.

Since a sequence in a metric space converges to a certain limit if and only if each subsequence has a subsequence which converges to that same limit, it suffices to prove $u_{n} \rightarrow u$ for a subsequence.

Since $\left(u_{n}\right)$ is bounded in $M R$ by the estimate (4.7), and since $M R$ is a Hilbert space, we may assume (after passing to a subsequence) that $u_{n} \rightarrow w$ in $M R$. For a subsequence, we may in addition assume that

$$
\begin{array}{cl}
\dot{u}_{n} \rightarrow \dot{w} & \text { in } L^{2}\left(0, T ; L^{2}(\Omega)\right), \text { and } \\
A u_{n} \rightarrow A w & \text { in } L^{2}\left(0, T ; L^{2}(\Omega)\right) .
\end{array}
$$

We show that $w=u$. Since $v_{n} \rightarrow v$ in $E$, we may assume (after passing to a subsequence again) that there exists a function $h_{k} \in L^{2}\left((0, T) \times \Omega_{k}\right)$ such that

$$
\begin{aligned}
& \left(v_{n}, \nabla v_{n}\right) \rightarrow(v, \nabla v) \text { almost everywhere on }(0, T) \times \Omega \text { and } \\
& \left|v_{n}\right|+\left|\nabla v_{n}\right| \leq h_{k} \text { almost everywhere on }(0, T) \times \Omega_{k}, \text { for every } n \in \mathbb{N} .
\end{aligned}
$$

The almost everywhere convergence on $(0, T) \times \Omega$ is established by using a diagonalization argument. 
Then, by the continuity of $\beta$ and $f$,

$$
\begin{aligned}
m_{v_{n}}(t, x):=\beta\left(t, x, v_{n}, \nabla v_{n}\right) \rightarrow & \beta(t, x, v, \nabla v)=: m_{v}(t, x) \text { and } \\
f_{v_{n}, k}(t, x):=f\left(t, x, v_{n}, \nabla v_{n}\right) 1_{\Omega_{k}}(x) \rightarrow & f(t, x, v, \nabla v) 1_{\Omega_{k}}(x)=: f_{v, k}(t, x) \\
& \text { almost everywhere on }(0, T) \times \Omega .
\end{aligned}
$$

Moreover, by the growth assumption on $f$ and the uniform domination of $v_{n}$, we have

$$
\left|f_{v_{n}, k}\right| \leq g+L h_{k} \quad \text { almost everywhere on }(0, T) \times \Omega_{k}, \text { for every } n \in \mathbb{N} .
$$

Recall that, for every $n \in \mathbb{N}$,

$$
\dot{u}_{n}+m_{v_{n}} A u_{n}=f_{v_{n}, k} .
$$

By the dominated convergence theorem, $f_{v_{n}, k} \rightarrow f_{v, k}$ strongly (and weakly) in $L^{2}\left(0, T ; L^{2}(\Omega)\right)$. Moreover, by the dominated convergence theorem, for every $\varphi \in$ $L^{2}\left(0, T ; L^{2}(\Omega)\right)$,

$$
m_{v_{n}} \varphi \rightarrow m_{v} \varphi \text { in } L^{2}((0, T) \times \Omega) .
$$

Since $A u_{n} \rightarrow A w$ in $L^{2}\left(0, T ; L^{2}(\Omega)\right)$, it follows that for every $\varphi \in L^{2}\left(0, T ; L^{2}(\Omega)\right)$

$$
\int_{0}^{T} \int_{\Omega} m_{v_{n}} A u_{n} \varphi \rightarrow \int_{0}^{T} \int_{\Omega} m_{v} A w \varphi
$$

or, in other words,

$$
m_{v_{n}} A u_{n} \rightarrow m_{v} A w \quad \text { in } L^{2}((0, T) \times \Omega) .
$$

Thus, letting $n \rightarrow \infty$ in (4.8) shows that

$$
\dot{w}(t)+m_{v} A w(t)=f_{v, k}(t) \quad \text { for almost every } t \in(0, T) .
$$

Since $M R \hookrightarrow C([0, T] ; V)$, we have also $u_{n} \rightarrow w$ in $C([0, T] ; V)$ and in particular $w(0)=w-\lim _{n \rightarrow \infty} u_{n}(0)=u_{0}$. Since also $u$ is solution of the problem $\dot{u}(t)+m_{v} A u(t)=f_{v, k}(t)$ and $u(0)=u_{0}$, and since the solution of this problem is unique by Theorem 3.2, this shows that $w=u$.

We have shown that $u_{n} \rightarrow u$ in $M R$. Since the embedding $M R \hookrightarrow E$ is compact, this implies that, after passing to a subsequence again, $u_{n} \rightarrow u$ in $E$. Therefore, $T_{k}$ is continuous.

(b) We prove that there exists a constant $c=c(\varepsilon, M, \eta, \omega, L, T) \geq 0$ independent of $k$ such that for every element $u$ in the Schaefer set

$$
\mathcal{S}_{k}=\left\{u \in E: u=\lambda T_{k} u \text { for some } \lambda \in[0,1]\right\}
$$

the estimate (4.6) holds. 
Assume that $u=\lambda T_{k} u$ for some $\lambda \in[0,1]$. Note that $u=\lambda T_{k} u$ if and only if

$$
\begin{aligned}
& \dot{u}(t)+m(t, \cdot, u, \nabla u) A u(t)=\lambda f(t, \cdot, u, \nabla u) 1_{\Omega_{k}} \text { for a.e. } t \in(0, T), \\
& u(0)=u_{0} .
\end{aligned}
$$

By multiplying the differential equation by $\frac{\dot{u}}{m_{u}}$ and integrating over $\Omega$, we obtain

$$
\begin{aligned}
\int_{\Omega} \dot{u}(t)^{2} \frac{d x}{m_{u}} & +\int_{\Omega} A u(t) \dot{u}(t) d x=\lambda \int_{\Omega_{k}} f(t, x, u, \nabla u) \dot{u} \frac{d x}{m_{u}} \\
& \leq \frac{1}{2} \int_{\Omega}|f(t, x, u, \nabla u)|^{2} \frac{d x}{m_{u}}+\frac{1}{2} \int_{\Omega} \dot{u}(t)^{2} \frac{d x}{m_{u}} \\
& \leq \int_{\Omega} g(t)^{2} \frac{d x}{m_{u}}+2 L^{2} \int_{\Omega}\left(|u(t)|^{2}+|\nabla u(t)|^{2}\right) \frac{d x}{m_{u}}+\frac{1}{2} \int_{\Omega} \dot{u}(t)^{2} \frac{d x}{m_{u}} .
\end{aligned}
$$

Using the estimate $\varepsilon \leq \frac{1}{m_{u}} \leq \frac{1}{\varepsilon}$ and the equality $\frac{1}{2} \frac{d}{d t} a(u(t))=(A u(t), \dot{u}(t))_{L^{2}}$, we thus obtain, for almost every $t \in[0, T]$,

$$
\frac{\varepsilon}{2} \int_{\Omega} \dot{u}(t)^{2}+\frac{1}{2} \frac{d}{d t} a(u(t))=\frac{1}{\varepsilon} \int_{\Omega} g(t)^{2}+\frac{2 L^{2}}{\varepsilon}\|u(t)\|_{V}^{2} .
$$

We integrate this inequality on $(0, t)$, use the boundedness and the ellipticity of the form $a$, and we obtain

$$
\begin{aligned}
\frac{\varepsilon}{2} \int_{0}^{t}\|\dot{u}(s)\|_{L^{2}}^{2} d s & +\frac{\eta}{2}\|u(t)\|_{V}^{2} \leq M\left\|u_{0}\right\|_{V}^{2}+\frac{1}{\varepsilon} \int_{0}^{t}\|g(s)\|_{L^{2}}^{2} d s \\
& +\frac{\omega}{2}\|u(t)\|_{L^{2}}^{2}+\frac{2 L^{2}}{\varepsilon} \int_{0}^{t}\|u(s)\|_{V}^{2} d s .
\end{aligned}
$$

As in (3.7), we can estimate the third term on the right-hand side of this inequality. It follows that

$$
\begin{aligned}
\frac{\varepsilon}{4} \int_{0}^{t}\|\dot{u}(s)\|_{L^{2}}^{2} d s+\frac{\eta}{2}\|u(t)\|_{V}^{2} \leq & \left(M+\frac{\omega}{2}\right)\left\|u_{0}\right\|_{V}^{2}+\frac{1}{\varepsilon}\|g\|_{L^{2}\left(0, T ; L^{2}(\Omega)\right)}^{2} \\
& +\left(\frac{\omega^{2}}{\varepsilon}+\frac{2 L^{2}}{\varepsilon}\right) \int_{0}^{t}\|u(s)\|_{V}^{2} d s
\end{aligned}
$$

This estimate is similar to the estimate (3.8) from the proof of Theorem 3.2. As in the proof of Theorem 3.2 we can now continue to estimate, and we see that there exists a constant $c=c(\varepsilon, M, \eta, \omega, L, T) \geq 0$ such that the estimate (4.6) is true for every $u \in \mathcal{S}_{k}$.

(c) In particular, the set $\mathcal{S}_{k}$ is bounded in $M R$. By continuity of the embedding (4.4) (or just a simple direct estimate of the corresponding norms), this implies that there exists $R>0$ such that $\mathcal{S}_{k}$ is included in

$$
\mathcal{C}_{k}:=\left\{v \in E: p_{k}(v)<R\right\} .
$$


It follows from the definition of $T_{k}$ and the estimate (4.7) that $T_{k} \mathcal{C}_{k}$ is contained in a bounded subset of $M R$. By compactness of the embedding (4.4), this implies that $T_{k} \mathcal{C}_{k}$ is contained in a compact subset of $E$.

Hence, by Schaefer's fixed point theorem (Theorem 2.2), the mapping $T_{k}$ admits a fixed point $u \in M R$. By the definition of $T_{k}$, this element $u$ is a solution of the problem (4.5) which, being an element of $\mathcal{S}_{k}$, satisfies the claimed estimate (4.6).

In the second step of the proof, we show that the problem (4.2) admits a solution. For every $k \in \mathbb{N}$, we choose a solution $u_{k}$ of the problem (4.5). Since every solution of the problem (4.5) is an element of $\mathcal{S}_{k}$ and satisfies the estimate (4.6) (which is independent of $k$ ), the sequence $\left(u_{k}\right)$ remains bounded in $M R$. Since $M R$ is a Hilbert space, we may therefore assume (after passing to a subsequence) that $u_{k} \rightarrow u$ in $M R$. Using the compactness of the embedding (4.4) and after passing to a subsequence again, if necessary, we may in addition assume that

$$
\begin{array}{ll}
\dot{u}_{k} \rightarrow \dot{u} & \text { in } L^{2}\left(0, T ; L^{2}(\Omega)\right), \\
A u_{k} \rightarrow A u & \text { in } L^{2}\left(0, T ; L^{2}(\Omega)\right), \\
\left(u_{k}, \nabla u_{k}\right) \rightarrow(u, \nabla u) \text { almost everywhere on }(0, T) \times \Omega \text { and } \\
\left|u_{k}\right|+\left|\nabla u_{k}\right| \leq h \text { almost everywhere on }(0, T) \times \Omega, \text { for every } k \in \mathbb{N},
\end{array}
$$

where $h \in L_{\text {loc }}^{2}((0, T) \times \Omega)$. The almost everywhere convergence and the domination may be proved by using a diagonalization argument.

By continuity of $\beta$ and $f$, and since $\Omega_{k}$ is increasing to $\Omega$, this implies

$$
\begin{aligned}
\beta\left(t, x, u_{k}, \nabla u_{k}\right) & \rightarrow \beta(t, x, u, \nabla u) \text { and } \\
f\left(t, x, u_{k}, \nabla u_{k}\right) 1_{\Omega_{k}}(x) & \rightarrow f(t, x, u, \nabla u) \text { almost everywhere on }(0, T) \times \Omega .
\end{aligned}
$$

By the growth assumption on $f$ and the domination of $u_{k}$, we have

$\left|f\left(t, x, u_{k}, \nabla u_{k}\right) 1_{\Omega_{k}}\right| \leq g+L h$ almost everywhere on $(0, T) \times \Omega$, for every $k \in \mathbb{N}$.

As in (4.9), this implies that

$$
\beta\left(t, x, u_{k}, \nabla u_{k}\right) A u_{k} \rightarrow \beta(t, x, u, \nabla u) A u \quad \text { in } L^{2}\left(0, T ; L^{2}(\Omega)\right) .
$$

As a consequence, we obtain that $f\left(t, x, u_{k}, \nabla u_{k}\right) 1_{\Omega_{k}}=\dot{u}_{k}+\beta\left(t, x, u_{k}, \nabla u_{k}\right) A u_{k}$ converges weakly in $L^{2}\left(0, T ; L^{2}(\Omega)\right)$. On the other hand, for every $\varphi \in L^{2}(0, T$; $\left.L^{2}(\Omega)\right)$ with compact support in $(0, T) \times \Omega$ we have

$$
\int_{0}^{T} \int_{\Omega} f\left(t, x, u_{k}, \nabla u_{k}\right) 1_{\Omega_{k}} \varphi \rightarrow \int_{0}^{T} \int_{\Omega} f(t, x, u, \nabla u) \varphi
$$

by the dominated convergence theorem. Since the compactly supported functions are dense in $L^{2}\left(0, T ; L^{2}(\Omega)\right)$, we thus obtain

$$
f\left(t, x, u_{k}, \nabla u_{k}\right) 1_{\Omega_{k}} \rightarrow f(t, x, u, \nabla u) \quad \text { in } L^{2}\left(0, T ; L^{2}(\Omega)\right) .
$$


Letting $k \rightarrow \infty$ in the problem (4.5), we therefore find that

$$
\dot{u}+\beta(t, x, u, \nabla u) A u=f(t, x, u, \nabla u) \quad \text { for a.e. } t \in(0, T) .
$$

We recall that $u_{k} \rightarrow u$ in $M R \hookrightarrow C([0, T] ; V)$ implies $u_{k}(0) \rightarrow u(0)$ in $V$, and therefore $u(0)=u_{0}$. Hence, $u$ is a solution to the problem (4.2). The estimate (4.3) for a solution of (4.2) is proved in a similar way than the a priori estimate of the set $\mathcal{S}_{k}$.

\section{Examples}

We now give several concrete examples.

Let $\Omega \subset \mathbb{R}^{d}$ be an open set. We denote by $\Delta_{\max }$ the maximal Laplacian on $L^{2}(\Omega)$, that is,

$$
\begin{aligned}
D\left(\Delta_{\max }\right) & :=\left\{u \in L^{2}(\Omega): \Delta u \in L^{2}(\Omega)\right\}, \\
\Delta_{\max } u & :=\Delta u .
\end{aligned}
$$

Then, by local regularity of the Laplacian, one has

$$
D\left(\Delta_{\max }\right) \subset H_{\mathrm{loc}}^{2}(\Omega) .
$$

Thus, condition (4.1) is satisfied whenever $A \subset \Delta_{\max }$, that is, whenever $A$ is a realization of the Laplacian with boundary conditions or, more generally, supplementary conditions. In order to apply Theorem 4.1, we also need to know that $A$ is selfadjoint and nonnegative. We give three examples of this type.

Example 5.1 (The Dirichlet-Laplacian). Let $V=H_{0}^{1}(\Omega)$ and $a(u, v)=\int_{\Omega} \nabla u \nabla v$. Let $A$ be the associated operator. Then $D(A)=H_{0}^{1}(\Omega) \cap D\left(\Delta_{\max }\right)$ and $A u=-\Delta u$ for every $u \in D(A)$.

Hence, if

$\beta$ and $f:(0, \infty) \times \Omega \times \mathbb{R}^{1+d} \rightarrow \mathbb{R}$ are measurable functions satisfying

the hypotheses of Theorem 4.1 on $(0, T) \times \Omega \times \mathbb{R}^{1+d}$ for every $T>0$

then, by Theorem 4.1, for every $u_{0} \in H_{0}^{1}(\Omega)$ the problem (1.1) from the Introduction admits a global solution

$$
u \in H_{\mathrm{loc}}^{1}\left([0, \infty) ; L^{2}(\Omega)\right) \cap L_{\mathrm{loc}}^{2}([0, \infty) ; D(A)) \cap C\left([0, \infty) ; H_{0}^{1}(\Omega)\right) .
$$

Example 5.2 (The Neumann-Laplacian). Let $u \in H^{1}(\Omega) \cap D\left(\Delta_{\max }\right)$. We say that $\frac{\partial u}{\partial v}=0$ weakly if $\int_{\Omega} \nabla u \nabla v+\int_{\Omega} \Delta u v=0$ for every $v \in H^{1}(\Omega)$. This is motivated by Green's formula

$$
\int_{\Omega} \nabla u \nabla v+\int_{\Omega} \Delta u v=\int_{\partial \Omega} \frac{\partial u}{\partial v} v d \sigma
$$

which is valid for $u \in C^{2}(\bar{\Omega}), v \in C^{1}(\bar{\Omega})$ and if $\Omega$ is bounded and of class $C^{1}$. 
Let $V=H^{1}(\Omega)$ and $a(u, v)=\int_{\Omega} \nabla u \nabla v$. Then the associated operator $A$ is given by

$$
\begin{aligned}
D(A) & =\left\{u \in H^{1}(\Omega) \cap D\left(\Delta_{\max }\right): \frac{\partial u}{\partial v}=0 \text { weakly }\right\}, \\
A u & =-\Delta u .
\end{aligned}
$$

Using this operator $A$ with the above interpretation of the homogeneous Neumann boundary condition, and if $\beta$ and $f$ are as in (5.2), then, by Theorem 4.1, for every $u_{0} \in H^{1}(\Omega)$, the problem

$$
\begin{cases}u_{t}-\beta(t, x, u, \nabla u) \Delta u=f(t, x, u, \nabla u) & \text { in }(0, \infty) \times \Omega, \\ \frac{\partial u}{\partial v}=0 & \text { in }(0, \infty) \times \partial \Omega, \\ u(0, \cdot)=u_{0}(\cdot) & \text { in } \Omega,\end{cases}
$$

admits a global solution

$$
u \in H_{\mathrm{loc}}^{1}\left([0, \infty) ; L^{2}(\Omega)\right) \cap L_{\mathrm{loc}}^{2}([0, \infty) ; D(A)) \cap C\left([0, \infty) ; H^{1}(\Omega)\right) .
$$

In this example, it is easy to see that one may in general not expect uniqueness of solutions. If $\Omega$ is bounded, and if one considers solutions $u$ which depend only on $t$ (that is, $u(t, \cdot)$ is constant on $\Omega$ ), then the problem (5.3) reduces essentially to an ordinary differential equation for which nonuniqueness is known. For example, if $f(t, x, u, p)=2 \sqrt{|u|}$ and if the initial value $u_{0}=0$, then $u(t, x)=0$ and $u(t, x)=t^{2}$ are two solutions of (5.3).

Example 5.3 (The Robin-Laplacian). Let $\Omega$ be bounded with Lipschitz boundary, and let $b \in C(\partial \Omega)$ be positive. Let $V=H^{1}(\Omega)$ and $a: V \times V \rightarrow \mathbb{R}$ be given by

$$
a(u, v)=\int_{\Omega} \nabla u \nabla v+\int_{\partial \Omega} b u v d \sigma,
$$

where $u$ and $v$ on the boundary are given by the trace operator, and $\sigma$ is the surface measure on $\partial \Omega$. Then $a$ is continuous, symmetric and $L^{2}(\Omega)$-elliptic. The associated operator $A$ is given by

$$
\begin{aligned}
D(A) & =\left\{u \in H^{1}(\Omega) \cap D\left(\Delta_{\max }\right): \frac{\partial u}{\partial v}+b u=0\right\}, \\
A u & =\Delta u .
\end{aligned}
$$

Here we use the following weak normal derivative. Let $h \in L^{2}(\partial \Omega), u \in H^{1}(\Omega) \cap$ $D\left(\Delta_{\max }\right)$. We say that $\frac{\partial u}{\partial v}=h$ if

$$
\int_{\Omega} \nabla u \nabla v+\int_{\Omega} \Delta u v=\int_{\partial \Omega} h v d \sigma \quad \text { for all } v \in H^{1}(\Omega) .
$$


Hence, if the functions $\beta$ and $f$ are as in (5.2), then, by Theorem 4.1, for every $u_{0} \in H^{1}(\Omega)$, the problem

$$
\begin{cases}u_{t}-\beta(t, x, u, \nabla u) \Delta u=f(t, x, u, \nabla u) & \text { in }(0, \infty) \times \Omega, \\ \frac{\partial u}{\partial v}+b u=0 & \text { in }(0, \infty) \times \partial \Omega, \\ u(0, \cdot)=u_{0}(\cdot) & \text { in } \Omega,\end{cases}
$$

admits a global solution

$$
u \in H_{\mathrm{loc}}^{1}\left([0, \infty) ; L^{2}(\Omega)\right) \cap L_{\mathrm{loc}}^{2}([0, \infty) ; D(A)) \cap C\left([0, \infty) ; H^{1}(\Omega)\right) .
$$

Example 5.4 (Elliptic operators). Let $a_{i j} \in C^{1}(\Omega) \cap L^{\infty}(\Omega)$ such that $a_{i j}=a_{j i}$ and

$$
\sum_{i, j} a_{i j}(x) \xi_{i} \xi_{j} \geq \alpha|\xi|^{2} \quad \text { for every } \xi \in \mathbb{R}^{d}, x \in \Omega .
$$

Let $V$ be a closed subspace of $H^{1}(\Omega)$ which is dense in $L^{2}(\Omega)$. Define $a: V \times V \rightarrow$ $\mathbb{R}$ by

$$
a(u, v)=\int_{\Omega} \sum_{i, j} a_{i j} \partial_{i} u \partial_{j} v .
$$

Then $a$ is symmetric, continuous and $L^{2}(\Omega)$-elliptic. Let $A$ be the operator associated with $a$ on $L^{2}(\Omega)$. Then for $u \in D(A)$ one has

$$
A u=\sum_{i, j} \partial_{j}\left(a_{i j} \partial_{i} u\right)
$$

in the sense of distributions. Hence, $D(A) \subset H_{\text {loc }}^{2}(\Omega)$ by [8, 6.3.1, Theorem 1] or [9, Theorem 8.9].

If we consider homogeneous Dirichlet boundary conditions (so that $V=H_{0}^{1}(\Omega)$ ), and if the functions $\beta$ and $f$ are as in (5.2), then Theorem 4.1 implies that, for every $u_{0} \in H_{0}^{1}(\Omega)$, the problem

$$
\begin{cases}u_{t}-\beta(t, x, u, \nabla u) \sum_{i, j} \partial_{j}\left(a_{i j} \partial_{i} u\right)=f(t, x, u, \nabla u) & \text { in }(0, \infty) \times \Omega, \\ u=0 & \text { in }(0, \infty) \times \partial \Omega \\ u(0, \cdot)=u_{0}(\cdot) & \text { in } \Omega,\end{cases}
$$

admits a global solution

$$
u \in H_{\mathrm{loc}}^{1}\left([0, \infty) ; L^{2}(\Omega)\right) \cap L_{\mathrm{loc}}^{2}([0, \infty) ; D(A)) \cap C\left([0, \infty) ; H_{0}^{1}(\Omega)\right) .
$$




\section{References}

[1] H. Amann, Maximal regularity and quasilinear parabolic boundary value problems, In: "Recent Advances in Elliptic and Parabolic Problems", World Sci. Publ., Hackensack, NJ, 2005, 1-17.

[2] H. Amann, Quasilinear parabolic problems via maximal regularity, Adv. Differential Equations 10 (2005), 1081-1110.

[3] H. Berestycki, L. Nirenberg, and S. R. S. Varadhan, The principal eigenvalue and maximum principle for second-order elliptic operators in general domains, Comm. Pure Appl. Math. 47 (1994), 47-92.

[4] Th. Cazenave and A. Haraux, "An Introduction to Semilinear Evolution Equations", Oxford Lecture Series in Mathematics and its Applications, Vol. 13, Oxford University Press, New York, 1998.

[5] Ph. ClÉment and S. LI, Abstract parabolic quasilinear problems and application to a groundwater flow problem, Adv. Math. Sci. Appl. 3 (1994), 17-32.

[6] R. DaUtray and J.-L. LiOns, "Analyse Mathématique et Calcul Numérique pour les Sciences et les Techniques", Vol. II, INSTN: Collection Enseignement, Masson, Paris, 1985.

[7] R. Dautray and J.-L. Lions, "Analyse Mathématique et Calcul Numérique pour les Sciences et les Techniques", Vol. VIII, INSTN: Collection Enseignement, Masson, Paris, 1987.

[8] L. C. Evans, "Partial Differential Equations", Graduate Studies in Mathematics, Vol. 19, American Mathematical Society, Providence, RI, 1998.

[9] D. Gilbarg and N. S. Trudinger, "Elliptic Partial Differential Equations of Second Order", Springer Verlag, Berlin, Heidelberg, New York, 2001.

[10] O. A. LADYŽEnSKaJa, V. A. SolonnikOV, and N. N. URAL'CEVA, "Linear and Quasilinear Equations of Parabolic Type", Translated from the Russian by S. Smith. Translations of Mathematical Monographs, Vol. 23, American Mathematical Society, Providence, R.I., 1967.

[11] G. M. Lieberman, "Second Order Parabolic Differential Equations", World Scientific Publishing Co. Inc., River Edge, NJ, 1996.

[12] A. LunARDI, "Analytic Semigroups and Optimal Regularity in Parabolic Problems", Progress in Nonlinear Differential Equations and Their Applications, Vol. 16, Birkhäuser, Basel, 1995.

[13] J. PRÜsS, Maximal regularity for evolution equations in $L_{p}$-spaces, Conf. Semin. Mat. Univ. Bari 285 (2002), 1-39.

[14] H. H. SCHAEFER, Über die Methode der a priori-Schranken, Math. Ann. 129 (1955), 415-416.

[15] R. E. Showalter, "Monotone Operators in Banach Space and Nonlinear Partial Differential Equations", Mathematical Surveys and Monographs, vol. 49, American Mathematical Society, Providence, RI, 1997.

[16] A. TyChOnOFF, Ein Fixpunktsatz, Math. Ann. 111 (1935), 767-776.

[17] A. Vitolo, Maximum principles for second-order parabolic equations, J. Partial Differential Equations, (4) 17 (2004), 289-302.

Institut für Angewandte Analysis

Universität Ulm

D-89069 Ulm, Germany

wolfgang.arendt@uni-ulm.de

Université Paul Verlaine - Metz

Laboratoire de Mathématiques et Applications de Metz et CNRS

UMR 7122, Bât. A, Ile du Saulcy 57045 Metz Cedex 1, France chill@univ-metz.fr 